\title{
A Novel Approach for Quantification of Retinal Vessel Tortuosity using Quadratic Polynomial Decomposition
}

\author{
Arunava Chakravarty \\ CVIT, IIIT Hyderabad \\ Hyderabad, India \\ Email: arunava.chakravarty@ research.iiit.ac.in
}

\author{
Jayanthi Sivaswamy \\ CVIT, IIIT Hyderabad \\ Hyderabad, India \\ Email: jsivaswamy@iiit.ac.in
}

\begin{abstract}
This paper describes a novel tortuosity measure, based on the premise that tortuosity is a measure of deviation from an ideal non-tortuous vessel. Hence, we propose to model the overall shape of an ideal vessel as a quadratic polynomial at a larger scale while the deviations are modeled as quadratic polynomials at smaller scales. Thus, a given vessel center-line is decomposed as a sum of quadratic polynomials of decreasing scale. This Quadratic Polynomial Decomposition is used as a framework for defining a quantitative measure of tortuosity. As opposed to the existing proposed measures, our method can distinguish between the relative size, shapes and orientations of the vessel bends. The measure is position and scale invariant and satisfies two key desired properties: it varies directly with frequency of twists at fixed amplitude and it varies directly with amplitude of twists when their frequency is fixed. The proposed method has been tested on a standard data set containing 30 artery and 30 vein vessel segments, and shown to be among the best measures as compared to the results of existing methods.
\end{abstract}

\section{INTRODUCTION}

Healthy Retinal Vessels are either straight or gently curved. In some diseases, vessels develop a number of twists and take on a serpentine path. Such vessels are termed as tortuous vessels. Tortuosity is one of the earliest changes in vessel morphology that occurs at the onset of many retinal and systemic deseases. The cause of tortuosity is attributed to stretching of the blood vessels due to increase in blood pressure or weak vessel walls [1]. Some of the common diseases, that may result in tortuous vessels are cardiovascular diseases such as hypertension [3] ,familial retinal arteriolar tortuosity [2], occlusion of retinal arteries and veins and diabetic Retinopathy [7]. It is the primary symptom used for tracking plus disease in Retinopathy of Prematurity [6]. Figures 1 and 2 illustrates the visual difference between a healthy and a tortuous vasculature network in retinal Color Fundus Images.

Tortuosity of vessels is graded by ophthalmologists qualitatively on a scale varying from 2 to 5 [1] based on their clinical judgment. The absence of any gold standard for grading vessel tortuosity makes it highly susceptible to intra and inter observer variability in grading. A robust tortuosity measure, is highly desirable in CAD systems as an aid in early detection and tracking the progress of diseases.

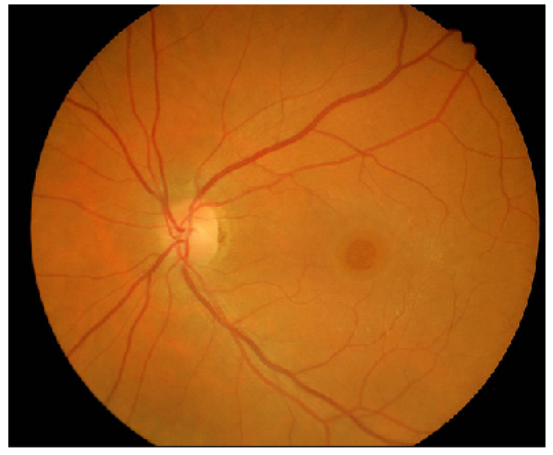

Fig. 1. Fundus Image of a healthy Eye

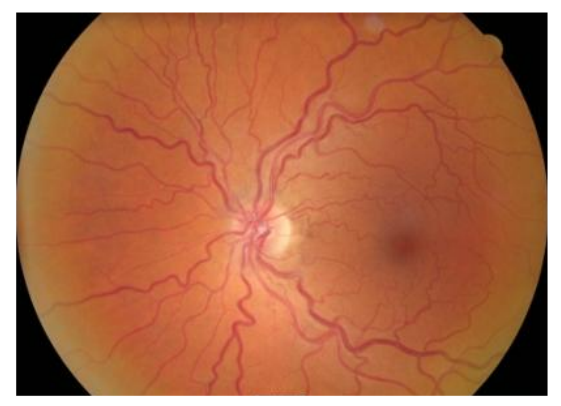

Fig. 2. Fundus Image having tortuous blood vessels

One of the earliest measures proposed was the Arc to Length Ratio [4], which used the ratio of actual length of the vessel to its chord length as a tortuosity measure. It was error prone as it failed to differentiate between vessels with gentle arc over large chord length and vessels with large number of twists. Dougherty and Varro [10], represented vessels as a 1D signal and proposed a measure based on the absolute sum of second derivatives of the signal normalized by the sampling interval. Six variants of curvature based measures were compared by Hart et al. [1] and two were identified to correlate well with clinical perception of tortuosity: the sum of squared curvature without normalization and the sum of curvature normalized by 
arc length. The main disadvantage of curvature based methods is that the results are highly dependent on the technique used in computation of curvature. Direct computation using finite differences do not give desirable results. Spline representation [5] or arbitrary smoothing [1] is required. A recursive formulation calls for bisection of chord lengths at each step and measuring the rate of increase of bisected chord lengths to the original chord length [6]. Another approach quantizes the vessels into discrete points and computes for each point, the average angle of line segments joining the previous and the next points [11]. This method is highly sensitive to noise and is dependent on the quantisation process. A recent approach divides the vessels into turn curves (vessel segments with constant sign of curvature) and defines tortuosity as a product of the sum of arc-chord ratio of each turn curve [5]. Included in the measure is a term to account for number of twists, normalized by chord length. Starting with the notion of an osculating circle at a point as the largest circle touching it and having maximum contact length with the centerline, a measure is proposed in [8] based on the average of the reciprocal of the radii of osculating circles over each vessel centerline pixel.

Thus, a common strategy is to divide the vessel centerline into constituent bends, quantify the tortuosity measure for each bend and take an average or sum of the tortuosity values of all the segments [4], [1], [5], [8]. Using measures such as the arc-chord ratio for quantifying curvature of each bend, the information related to the relative size of the bends is lost. Also, the orientation among the vessel bends is not given any consideration. However, both size and orientation have important effect on the shape of the vessel and neglecting them will lead to a loss of discrimination among a wide variety of vessels which are perceptually very different.

In this work, we propose an intuitive model for multi-scale representation of vessel segments. We refer to it as Quadratic Polynomial Decomposition (QPD). This permits comparison of tortuosity of vessels at multiple scales and takes into account the scale and relative orientation of the vessel bends. We also propose a quantitative measure of tortuosity based on the QPD framework. QPD is inspired from the observation that to determine tortuosity, experts have a mental reference of an ideal non-tortuous vessel for the given vessel and they measure significant deviations from it often in the form of sharp $U$ shaped bends to define tortuosity [7]. A multi-scale QPD framework provides an effective way of representing both the underlying ideal non-tortuous shape of vessel at a large scale as well as the local bends at smaller scales.

The rest of the paper is organised as follows: Section II presents a method for converting a vessel centerline to a 1D signal. Section III presents the details of the proposed QPD based on which a tortuosity measure is defined in Section IV. Section V verifies the basic abstract properties of the proposed tortuosity measure. Section VI, presents the details of assessment of the proposed measure on a publicly available dataset RET-TORT [5], followed by a discussion and conclusion.

\section{Converting Vessel Centerline to a 1D signal}

Similar to [5] we start with a set of vessel segments as input on which a set of points are marked by an expert on the vessel centerline. These points are interpolated first, using bicubic spline interpolation, to obtain the vessel centerlines. For rotation invariance, Principal Component Analysis is computed on the vessel centreline points following which each vessel centerline pixel is projected to their principal components. The direction of the first principle component is along the length of the vessel as the centerline pixels show maximum variance in this direction, while the second principle component lies perpendicular to it.It ensures that irrespective of the initial orientation of vessels, all centerline points are transformed to a coordinate system where $\mathrm{x}$-axis corresponds to the direction of length of the vessel and y-axis is perpendicular to it. Next we quantize the projected points along the principal axis and obtain the corresponding second principal component values as a 1D signal. Figure 3 illustrates a vessel segment, the interpolated centreline and the derived 1D signal.

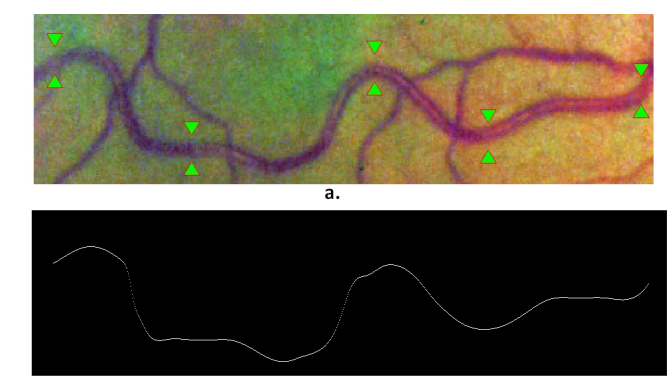

b.

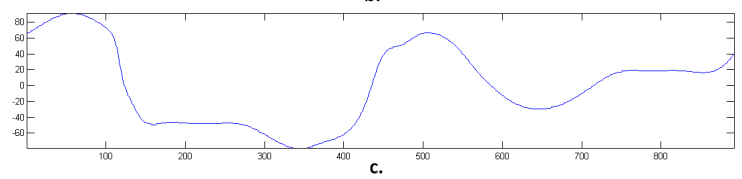

Fig. 3. a. An artery vessel segment from the RET-TORT dataset [5]; b. Centerline mask obtained by bicubic spline interpolation of points manually marked by an expert and c. Corresponding 1D signal

\section{Quadratic Polynomial Decomposition}

\section{A. Motivation}

We consider tortuosity of a given vessel as a measure which signifies the degree to which it deviates from an ideal nontortuous vessel. Since retinal images are projections of the spherical retinal surface on a 2D plane, even normal vessels appear to be gently curved in the image rather than following a straight path. Thus the reference is not a straight line but a curve with no twists (as twists contribute to tortuosity). Tortuosity is characterised by abnormal spring-like windings in vessels in 3 dimensions which project to ' $U^{\prime}$ or ' $S^{\prime}$ shaped bends in retinal images of varying shapes and sizes. Quadratic polynomials can offer a good approximation of such bends as polynomial fitting with a larger chord length (scale) can capture the underlying reference non-tortuous shape, while that of smaller scale fitted on deviation of the vessels from the reference smooth curve can be used to model the local 
bends. Thus quadratic polynomials offer a good model for representation of vessels at multiple scales.

\section{B. Methodology}

A 1D sequence representing a vessel segment centreline $V$,can be represented as a sum of localized quadratic polynomials, $P_{1}, P_{2}, \ldots . P_{n}$ defined at different scales. Each quadratic polynomial $P_{i}: y=a_{i} x^{2}+b_{i} x+c_{i}$ is defined by a 5-tuple $\left(a_{i}, b_{i}, c_{i}, s_{i}, l_{i}\right)$, where $s_{i}, l_{i}$ represent the starting position and chord length of $P_{i}$ respectively. Given a vessel segment $\mathrm{V}$ $\left\{v_{1}, v_{2}, \ldots v_{n}\right\}$, the steps in its quadratic polynomial decomposition (QPD) are as follows :

1) Find second order polynomial $\mathrm{P}\left\{p_{1}, p_{2}, \ldots p_{n}\right\}$ that fits the vessel $\mathrm{V}$ with least squared error. $\mathrm{P}$ is the quadratic polynomial approximation of $\mathrm{V}$ at current level.

2) Evaluate the Error signal, $E=\left(p_{1}-v_{1}\right),\left(p_{2}-v_{2}\right), \ldots,\left(p_{n}-v_{n}\right)$.

$E$ quantifies the deviation of the vessel $\mathrm{V}$ from the reference polynomial $\mathrm{P}$.

3) Compute the Error per unit length, $\varepsilon=\frac{\sum_{i=1}^{n} E(i)}{l}$, where $l$ represents the length of chord from $p_{1}$ to $p_{n}$.

4) Divide $E$ into sub-segments $E_{1}, E_{2} \ldots E_{n}$ demarcated by zero crossings of $E$.

5) If $\varepsilon<\delta$ then terminate the recursion. $\delta$ is a small tolerance, such that when $\varepsilon$ of a segment goes below it, the segment is assumed to be well represented. Otherwise, recursively apply steps 1 to 4 on each of the error signal segments $E_{1}, E_{2} \ldots E_{n}$ independently to obtain the decomposition at the next level.

Figure 4, shows the result of QPD of a vessel segment using the above method. The sample corresponds to the vessel segment "14_Arteria.jpg" from the RET-TORT dataset. Figure $4 \mathrm{~b}-\mathrm{d}$ represent the decomposition of the vessel at levels 1-3 respectively. The QPD allows perfect reconstruction implying that no information of the vessel shape is lost during this process. Figure $4 \mathrm{e}$, shows the result of reconstruction by addition of all the localized quadratic polynomials extracted at the different scales. Ideally, there should be zero error in reconstruction, however due to the use of an error tolerance $\delta=3$, we can observe some artifacts in the reconstruction.

\section{TORTUOSITY MEASURE}

We next show how the QPD framework can be used to derive a measure for tortuosity. Let the QPD of a vessel segment $\mathrm{V}$ be $P_{1}, P_{2}, . . P_{n}$ at different levels. We model the tortuosity measure $\tau(V)$ as a sum of contributions of each of these polynomials $P_{i}$ to the overall tortuosity. Let us denote these contributions by $\psi\left(P_{i}\right)$.

$$
\tau(v)=\sum_{i=1}^{n} \psi\left(P_{i}\right)
$$

Our aim is now to find the required characteristics of the function $\psi(P)$. Consider the four sample vessel segments A, $\mathrm{B}, \mathrm{C}, \mathrm{D}$ (in cyan) in figure 5 along with their quadratic polynomial decomposition (shown in red,magneta and green). Vessels $\mathrm{A}$ and $\mathrm{B}$ are decomposed into single quadratic polynomials $P_{a} ; P_{b}$ respectively at the first level of Quadratic Polynomial

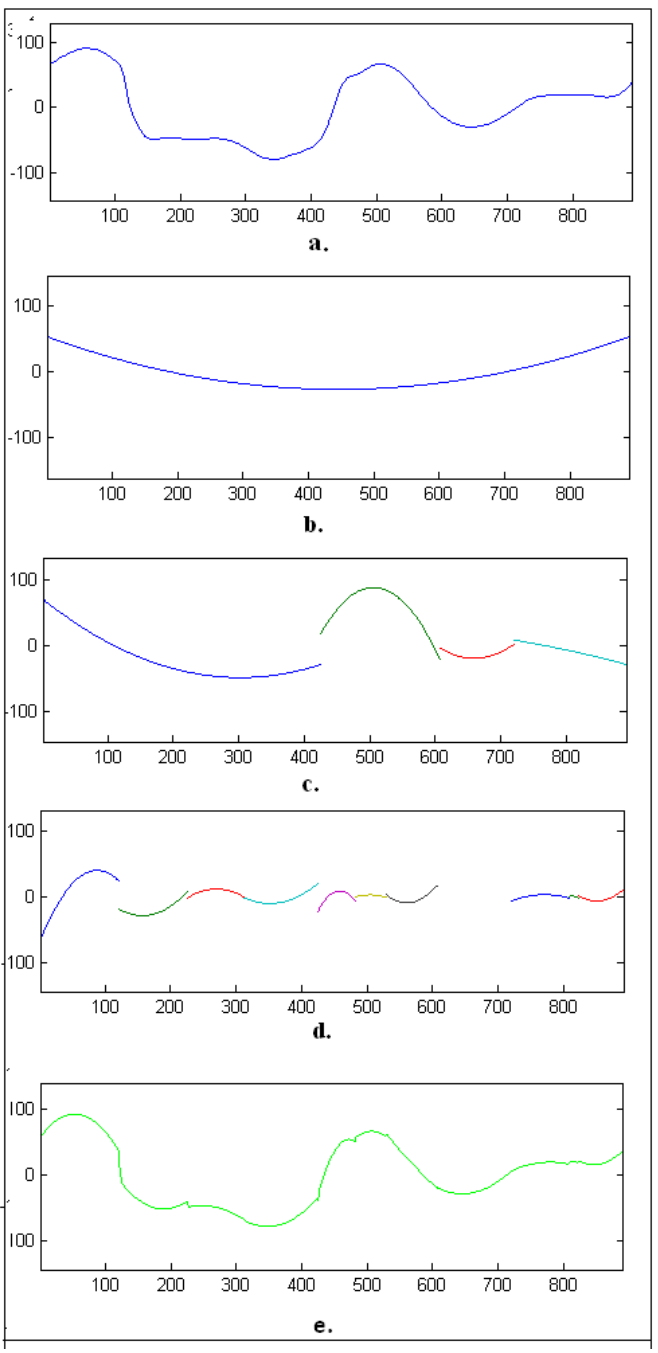

Fig. 4. Quadratic Polynomial Decomposition vessel: a. Sample vessel sequence, b. Quadratic polynomial obtained at scale 1, c. Set of Quadratic polynomials obtained at Scale 2, d. Set of quadratic polynomials obtained at scale 3 and e. Reconstructed image by addition of all the polynomials extracted at different scales.

Decomposition, while the vessels $\mathrm{C}$ is decomposed into 3 polynomials at 2 levels and Vessel D is decomposed into 7 polynomials at three levels.

Let us compare the trivial cases of vessels A and B. Both of them are approximated by single quadratic polynomials $P_{a}$ and $P_{b} ; P_{b}$ appears to be more curved than $P_{a}$. Nevertheless, both vessel segments are normal and must be assigned a tortuosity measure close to 0 . However, as we have to assign a relative quantitative measure to both the vessel segments, the measure must be slightly higher for $P_{b}$ than $P_{a}$. Thus, $\psi\left(P_{b}\right)>\psi\left(P_{a}\right)$, while both $\psi\left(P_{a}\right), \psi\left(P_{b}\right)$ are close to zero.

Comparing vessels $\mathrm{A}$ and $\mathrm{C}$, both have identical polynomial decomposition $P_{a}$ and $P_{c}$ at level 1 , but $\mathrm{C}$ requires more polynomials $P_{c 2}$ and $P_{c 3}$ at successive level for proper representation as compared to A. Clearly $\mathrm{C}$ is more tortuous than vessel A. This justifies the criterion $\psi\left(P_{i}\right)>0 \forall i$.

Comparing vessels $\mathrm{B}$ and $\mathrm{C}$ leads to an important observa- 


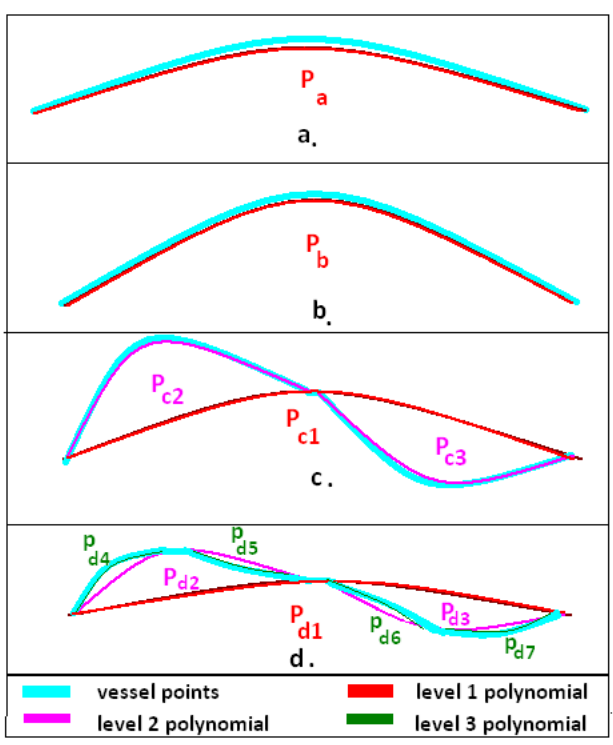

Fig. 5. Four sample vessel segments and their corresponding levels of Quadratic Polynomial Decomposition

tion. At level 1 , the vessels are represented by $P_{b}$ and $P_{c 1}$ respectively, with the former being more curved than the latter. Thus, $\psi\left(P_{c 1}\right)<\psi\left(P_{b}\right)$, and both $\psi\left(P_{c 1}\right), \psi\left(P_{b}\right)$ must be close to zero. However, since vessel $\mathrm{C}$ has bends at the successive higher levels of QPD, the overall tortuosity of vessel C should be greater.If $\psi\left(P_{c 2}\right), \psi\left(P_{c 3}\right)$, is greater than $\psi\left(P_{b}\right)$, then this condition is satisfied. This leads us to the conclusion that (i) for 2 segments of similar chord length, the segment with higher curvature is more tortuous and (ii) for 2 segments of different chord length, the segment with smaller chord length must be given a higher weight. Thus, $\psi(P)$ must be directly proportional to the curvature of the polynomial and inversely proportional to the underlying chord length.

It is worth noting that since the polynomials are based on fitting the error sequence rather than the original sequence, the curvature actually relates to the deviation from the underlying quadratic polynomial rather than a straight line.

Now, consider the vessels $\mathrm{C}$ and $\mathrm{D}$. At the first level, $P_{c 1}$ is more curved than $P_{d 1}$; at level 2 also $P_{c 2}$ and $P_{c 3}$ are more curved than $P_{d 2}$ and $P_{d 3}$. Considering the contributions of the polynomials only upto second level of decomposition, $\mathrm{C}$ is more tortuous than D. However,their relative tortuosity depends on the the contribution of polynomials at third level of decomposition $P_{d 4}-P_{d 7}$ for vessel D. If bends $P_{d 4}-P_{d 7}$ are perceptually significant, then vessel $\mathrm{D}$ will be more tortuous, however, if $P_{d 4}-P_{d 7}$ contributes insignificantly to the shape of the underlying polynomials at level 2 , they must be neglected. This is handled in the following two ways.Firstly, $\psi(P)$ being proportional to curvature of polynomial, $P_{d 4}$ to $P_{d 7}$ will have low contribution in tortuosity if they are perceptually insignificant. Secondly, Instead of exact decomposition, we have incorporated a tolerance $\delta$ while computing QPD on the amount of error that is allowed in the decomposition process. When error becomes below $\delta$, we consider the error to be perceptually insignificant and choose to ignore it. The vessel segments being made up of discrete pixels is not smooth but zigzag in nature.The threshld negates the impact of such aliasing effects and also improves the computational efficiency as number of levels of decomposition is reduced. An additional constraint on $\psi(P)$ is that it should be normalized by the overall length of the vessel, so that though we can use $\psi$ to compare the relative size of the bends in the same vessel, the measure should be invariant to differences in the overall size of vessel segments.

Proposed Tortuosity Measure: We define $\psi(P))=\frac{\operatorname{Area}(P)}{l^{2} \times L}$ as the desired toruosity measure. $\frac{\operatorname{Area}(P)}{l}$ which is defined below, provides a measure of curvature of the vessel bend; $L$ represents the chord length of the entire vessel segment and helps normalize by the scale of the entire vessel segment and allow comparison of tortuosity of vessel segments of different scale; an additional term $l$ serves to normalize the measure by its chord length to give higher weightage to local bends. The numerator of $\psi$ is computed as follows:

$$
\begin{array}{r}
\operatorname{Area}\left(P_{i}\left(a_{i}, b_{i}, c_{i}, s_{i}, l_{i}\right)\right)=\int_{1}^{l}\left(a_{i} x^{2}+b_{i} x+c_{i}\right) d x \\
=\frac{a_{i}}{3} \times\left(l^{3}-1\right)+\frac{b_{i}}{2} \times\left(l^{2}-1\right)+c_{i} \times(l-1)
\end{array}
$$

To summarize, given a $1 \mathrm{D}$ sequence representing a vessel segment centerline, with $A$ being the Area of the least square quadratic polynomial $P$ fit to the error segment of length $l$ on $\mathrm{V}, L$ the chord length of $\mathrm{V}$, the tortuosity $\tau(V)$ is defined as

$$
\tau(V)= \begin{cases}\left\{\frac{A}{L \times l^{2}}\right\} & \text { if Error } \leq \delta \\ \left\{\frac{A}{L \times l^{2}}\right\}+\sum_{i=1}^{n} \tau\left(E_{i}\right) & \text { Otherwise }\end{cases}
$$

In the above equation, $l=L$ at the first level of recursion. At successive levels, $L$ denotes the length of the entire vessel segment $\mathrm{V}$ while $l$ denotes the length of the error segments $E_{i}$ which are computed using QDP. Instead of computing QDP separartely and then applying equation 3 , we can combine the two steps into a simple recursive algorithm as described in algorithm 1. Steps 6-8 in Algorithm 1 correspond to the base condition of equation 3 ; steps $11-14$ computes the recursive definition, while remaining steps implements QPD.

\section{Abstract Properties}

Since, tortuosity of vessels is graded qualitatively by opthalmologists based on their clinical judgement, it is not possible to come up with a set of sufficient conditions for a good tortuosity measure. However, related works such as [1], [5], and [9] have discussed some intuitive features that may act as necessary conditions for any clinically meaningful tortuosity measure.

1) Invariance to Translation and Rotation: The measure is translation invariant as the Area, chord length of the quadratic polynomials, and the overall length of the vessel segment are translation invariant measures. Rotation invariance is achieved during the the process of converting the vessel centerline pixels to a 1D signal as discussed in section II. Since vessel centerline points are projected in the direction 


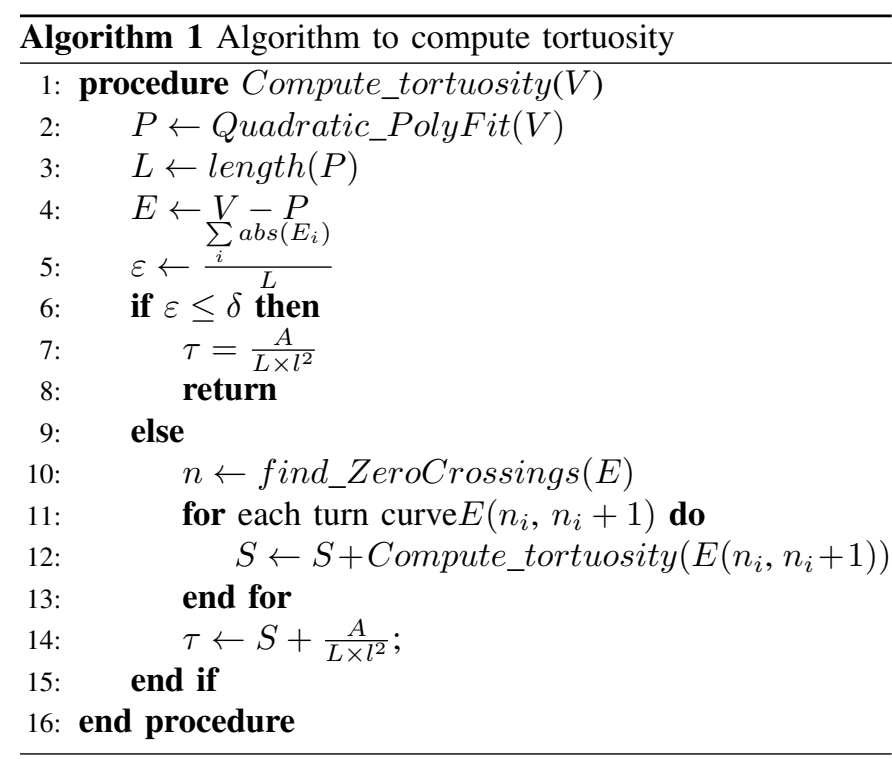

of their Principal Components,identical vessels with different orientation always map to the same 1D signal and hence would compute to having identical tortuosity measure.

2) Frequency Modulation: Given two vessels with identical amplitude but with different number of twists, tortuosity is directly proportional to the frequency of the twists [5]. Our measure conforms to this principle and we illustrate this property using vessel segments modeled as sinusoids of 3 increasing frequencies in figure 6. The computed tortuosity value increases correspondingly.

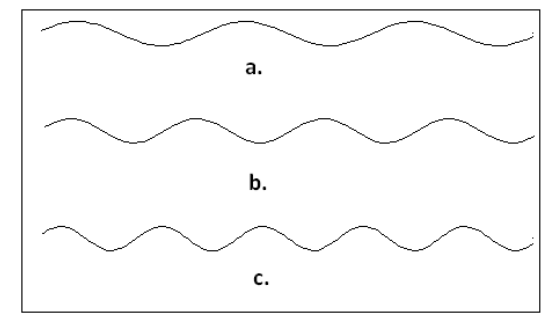

Fig. 6. Tortuosity dependency on frequency modulation. Vessel segments modeled as a. $\sin (3 \times t)$ b. $\sin (4 \times t)$ and c. $\sin (5 \times t)$. The computed tortuosity values are $\tau=0.0012,0.0022$ and 0.0035 for a,b,c respectively.

3) Amplitude Modulation: Given 2 vessels having equal number of twists but different amplitudes, tortuosity is directly proportional to the amplitude of the twists [5]. Our measure conforms to this principle and we illustrate this property using vessel segments modeled as sinusoids of 3 increasing amplitudes in figure 7 . The computed tortuosity values can be seen to increase correspondingly.

4) Scale Invariance: For tortuosity measure to be scale invariant, it must be normalized by the total chord length of the entire vessel resulting in a dimensionality of $L^{-1}$. The proposed measure satisfies this condition. For any vessel segment, the proposed tortuosity measure (eq.3) will be of form:

$$
\tau(v)=\sum_{i=1}^{n} \frac{A_{i}}{L \times l_{i}^{2}}=\frac{1}{L} \times \sum_{i=1}^{n} \frac{A_{i}}{l_{i}^{2}}
$$

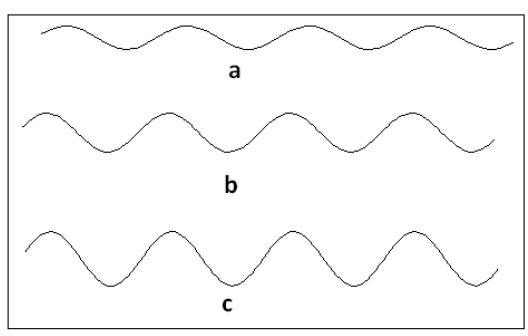

Fig. 7. Tortuosity dependency on amplitude modulation. Vessel segments modeled as a. $3 \times \sin (4 \times t)$,b. $5 \times \sin (4 \times t)$ and c. $7 \times \sin (4 \times t)$. The computed tortuosity values are $\tau=0.0022,0.0036$ and 0.0072 for a,b,c respectively.

where $\mathrm{n}$ is the total number of polynomials extracted at all scales. $\frac{1}{L}$ can be taken out as a common factor from all the summation terms, while the remaining summation terms of form: $\frac{A_{i}}{l_{i}^{2}}$ is a dimensionless quantity since both the numerator and denominaor are of same dimension $\left(L^{2}\right)$. Thus the overall dimension of the measure is $L^{-1}$.

\section{EXPERIMENTAL RESULTS}

We have evaluated our proposed method on a publicly available dataset RET-TORT [5]. RET-TORT dataset consists of 30 artery and 30 vessel segments of patients suffering from hypertension, manually marked and rank ordered by increasing degree of tortuosity, by an expert. Table I compares the Spearman rank correlations of the existing methods reported in literature by Grisan et al. [5], and our proposed method. Spearmann Rank coefficient gives a measure of how close

TABLE I

Comparative Results on RET-TORT Dataset: Performance of OTHER METHODS FROM [5]

\begin{tabular}{|l|l|l|}
\hline Method & Arteries & Veins \\
\hline$L_{c} / L_{x}$ & .792 & .656 \\
\hline$t_{c}$ & .922 & .837 \\
\hline$t_{s c}$ & .925 & .826 \\
\hline$t_{c} / L_{c}$ & .919 & .814 \\
\hline$t_{s c} / L_{c}$ & .917 & .773 \\
\hline$t_{c} / L_{x}$ & .939 & .842 \\
\hline$t_{s c} / L_{x}$ & .928 & .804 \\
\hline MAC & .920 & .814 \\
\hline TN & .838 & .695 \\
\hline ICM & .684 & .575 \\
\hline DCI & .787 & .589 \\
\hline Grisan et al. $[5]$ & .949 & .853 \\
\hline Proposed & .944 & $\mathbf{. 8 2 8}$ \\
Method & & \\
\hline
\end{tabular}

the tortuosity measure agrees to the relative ordering of the vessel segments by the expert. The first seven methods in this Table define tortuosity as Arc-Chord Ratio $\left(L_{c} / L_{x}\right)$, Integral of Absolute Curvature $\left(t_{c}\right)$, Integral of squared Curvature $\left(t_{s c}\right)$, Integral of Absolute Curvature normalized by chord length $\left(t_{c} / L_{c}\right)$, Integral of squared curvature normalized by chord length $\left(t_{s c} / L_{c}\right)$, Integral of Absolute Curvature Normalized by Arc length $\left(t_{c} / L_{x}\right)$, Integral of squared curvature normalized by chord length $\left(t_{s c} / L_{x}\right)$, respectively. The remaining measures are Mean Direction Angle Change $(M A C)$ [11], Absolute Direction Angle Change( $T N)$ [13], Inflection Count Metrics( $I C M)$ [14] and Absolute Direction Angle 
Change $(D C I)$ [12].The penultimate entry in the table is the result from [5]. They propose a measure $T$ that is dependent on 3 parameters: the chord length of the entire vessel segment $L_{c}$, number of turn curves in the vessel segment $n$ and the Arc-chord ratio of individual turn curves (vessel sub-segments with the same sign of curvature). Tortuosity $T$ is defined [5] as

$$
T(s)=\frac{n-1}{n} \times \frac{1}{L_{c}} \sum \frac{L_{c s i}}{L_{x s i}}-1
$$

From the tabulated results it can be seen that our proposed measure gives a Spearman rank correlation coefficient of 0.944 in the case of arteries and 0.828 for veins with respect to the expert ordering. The proposed method correlates well to the clinical perception of tortuosity and outperforms most of the existing methods. In comparison, with the best performing method [5] our results are identical up to second degree of decimal ( 0.944 vs 0.949$)$, while our performance is slightly below their performance for veins (0.828 vs 0.853$)$ [5]. However, the proposed method has a key advantage. We illustrate this with the help of an example. Figure 8 represents 3 synthetic vessel

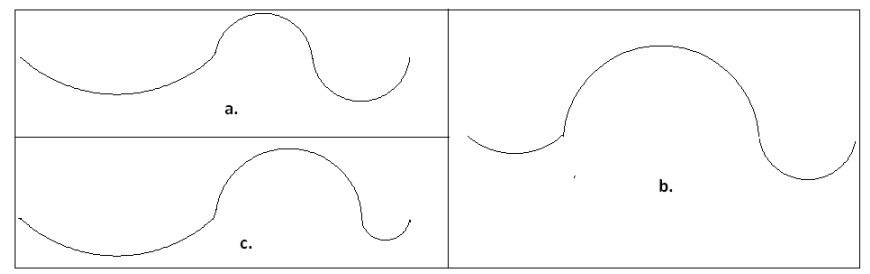

Fig. 8. Importance of scale: $a, b, c$ are synthetic vessel segments where corresponding turn curves have same arc-chord ratio but varying scale

segments with identical chord length over the entire vessel segment and 3 twists (turns).The arc to chord ratio of the 3 corresponding twists across all the 3 cases are same though the magnitude of arc and chord lengths vary greatly. The existing tortuosity metrics will yield identical values for these 3 cases though they are perceptually different. In contrast, the proposed measure will be able to distinguish between them since it takes into account the relative size of each turn curve and their relative orientation into account while computing tortuosity.

\section{CONCLUSION AND FUTURE WORK}

In this paper, we have proposed a novel tortuosity measure, which works on the underlying principle of QPD which helps decompose any curve into a set of quadratic polynomials at decreasing scales. The approach is based on the observation that experts quantify tortuosity based on a vessel's deviation from a reference normal vessel that best fits it. In practise, the deviations from the model vessel that leads to tortuosity is in the form of ' $U$ ' shaped bends which can be described by quadratic polynomials at a smaller scale.

We have shown that the proposed method conforms to the existing set of guidelines for a clinically meaningful tortuosity measure and additionally, takes into account the relative shape and to some extent the relative orientation of the vessel bends which have been neglected in the existing methods.
Though the underlying assumptions of our work have been intuitive and considers vessel bends at different relative scales and orientations, further investigation on a larger dataset with grading from multiple experts is necessary. Another important direction is to extend the model to take into account the vessel caliber as it also plays an important role in computation of tortuosity. Wide vessels in general have thicker walls, and hence even a small bend in a thick vessel may characterize an abnormal tortuous vessel, while more bend should be necessary to characterise tortuosity of thin vessels.

\section{ACKNOWLEDGMENT}

This work was supported partly by the Department of Science and Technology, Govt. of India, under Grant DST/INT/NL/Biomed/P(3)/2011(G).

The authors wish to thank Dr. S. Piermarocchi, from the Department of Ophthalmology, University of Padova, Italy, for having kindly provided the fundus images and the manual tortuosity grading.

\section{REFERENCES}

[1] W. E. Hart, M. Goldbaum, B. Cote, and P. Kube, "Measurement and classication of retinal vascular tortuosity," Int. J. Med. Informatics, vol. 53, no. 23, pp. 239-52, Feb. 1999.

[2] F. Sutter and H. Helbig, "Familial retinal arteriolar tortuosity: A review," Surv. Ophthalmol., vol. 48, no. 3, pp. 245-255, 2003.

[3] A. Stanton, B.Wasan, A. Cerutti, S. Ford, R.Marsh, P. Sever, S. Thom, and A. Hughes, "Vascular network changes in the retina with age and hypertension, ", J. Hypertension, vol. 13, no. 12, pp. 1724-1728, 1995.

[4] W. Lotmar, A. Freiburghaus, and D. Bracher, "Measurement of vessel tortuosity on fundus photographs," Graefes Arch. Clin. Exp. Ophthalmol, vol. 211, pp. 49-57, 1979.

[5] Enrico Grisan, Marco Foracchia, and Alfredo Ruggeri, "A Novel Method for the Automatic Grading of Retinal Vessel Tortuosity, " IEEE Transactions on Medical Imaging, vol.27, no.3, pp.310-319, March 2008

[6] Clare M. Wilson,Kenneth D. Cocker,Merrick J. Moseley,Carl Paterson,Simon T. Clay,William E. Schulenburg,Monte D. Mills,Anna L. Ells,Kim H. Parker,Graham E. Quinn,Alistair R. Fielder,and Jeffrey $\mathrm{Ng}$, "Computerized Analysis of Retinal Vessel Width and Tortuosity in Premature Infants," Invest Ophthalmol Vis Sci.,2008 Aug;49(8):3577-85.

[7] Divyanjali Satyarthi, B.A.N. Raju, and S. Dandapat, "Detection of Diabetic Retinopathy in Fundus Images using Vector Quantization Technique," India Conference, 2006 Annual IEEE,.

[8] C. Sinthanayothin, P. Panitsuk, B. Uyyanonvara, "Automatic retinal vessel tortuosity measurement," Nat. Electron. and Comput. Technol. Center, Nat. Sci. and Technol. Dev. Agency (NSTDA), Pathumthani, Thailand ,2010

[9] Rashmi Tudor ,Danu onkae Toshiaki Kondo, Bunyarit Uyyanonvara, ”A novel approach for quantification of retinal vessel tortuosity based on principal component analysis," The 8th Electrical Engineering/,Electronics, Computer, Telecommunications and Information Technology, Association of Thailand - Conference 2011.

[10] G.Dougherty and J.Varro, "A quantitative index for measurement of the tortuosity of blood vessels," Medical Engineering and Physics, pp 567574 , vol 22,2000 .

[11] K. V. Chandrinos,M. Pilu, R. B. Fisher, and P. Trahanias , "Image processing techniques for the quantication of atherosclerotic changes," presented at the MEDICON98, Limassol, Cyprus, Jun. 1998.

[12] M. Patasius, V. Marozas, D. Jegelevicius, and A. Lukosevicius, "Evaluation of tortuosity of eye blood vessels using the integral of square of derivative of curvature," IFMBE Proc. 3rd Eur. Med. Biol. Eng. Conf. (EMBEC05) , 2005, vol. 11.

[13] K. G. Goh, H. Wynne, M. L. Lee, and H. Wang, K. Cios, Ed., "Adris: An automatic diabetic retinal image screening system," Med. Data Min. Knowledge Discovery , 2001, pp. 181-210.

[14] E. Bullitt, G. Gerig, S. M. Pizer, W. Lin, and S. R. Aylward ,"Measuring tortuosity of the intracerebral vasculature from mra image," Med. Image Anal. , no. 9, pp. 1163-1171, Sep. 2003. 